\title{
Une enzyme comme auto-antigène dans la maladie d'Addison
}

La maladie d'Addison est due à une insuffisance surrénale qui associe des signes de déficit en corticostéroïdes (asthénie, anomalies du métabolisme glucidique et hydrosalin) à une pigmentation "bronzée " de la peau, attribuée à une hypersécrétion réactionnelle d'ACTH. Elle se présente tantôt comme un syndrome isolé, tantôt associée à d'autres endocrinopathies, et reconnaît alors une origine autoimmune. Deux types principaux de telles associations ont été décrits [1]. Le type 1, syndrome d'auto-immunité polyendocrinienne, est rare et frappe les enfants ; il débute le plus souvent par une atteinte des parathyroïdes, la maladie d'Addison se manifestant quelques années plus tard. Le type 2, plus fréquent, d'apparition plus tardive, associe la maladie d'Addison à une autre affection, hyper- ou hypothyroïdie, diabète de type 1 , déficit ovarien. En principe, le type 1 reconnaît une hérédité autosomique récessive [2], le type 2 est dominant et associé à HLA-DR3 [3].

Des auto-anticorps anti-surrénales ont déjà été mis en évidence dans la maladie d'Addison; les uns, surtout dans le type 1 , ont des réactions croisées avec des extraits d'autres glandes endocrines, d'autres non. On n'en avait encore jamais identifié la nature, contrairement à ce que l'on a récemment découvert dans d'autres endocrinopathies (Tableau I). Cette lacune vient d'être comblée grâce aux travaux de chercheurs finlandais et estoniens. Krohn et al. [4] ont préparé une banque d'expression d'ADNc de surrénales et examiné si des protéines exprimées par des clones issus de cette banque étaient reconnues par le sérum de malades. Ceux-ci étaient pour l'essentiel 35 enfants atteints du type 1 polyendocrinien, dont 28 avec des symptômes de maladie d'Addison, plus deux adultes porteurs de maladie d'Addison isolée. La banque a été préparée à partir d'ARN messager de surrénales d'un fœtus humain de 20 semaines. Les antigènes surrénaliens furent obtenus à partir d'organes prélevés sur des donneurs de reins post-mortem.

Les sérums de 21 des 35 sujets à syndrome endocrinien multiple donnèrent des précipités avec les extraits surrénaliens. Après électrophorèse et immunorévélation on trouvait cinq bandes s'étageant de 55 à $16 \mathrm{kDa}$. Cependant, en séquençant les clones immunoréactifs issus de la banque, on eut la surprise de voir que, sur les 39 clones testés, 38 présentaient la même séquence, identique à $99 \%$ à celle du gène de la cytochrome
P 450-stéroïde $17 \alpha$-hydroxylase ; la seule différence résidait dans une taille inégale des clones. Pour vérifier que les anticorps des malades réagissaient bien avec l'enzyme, un des fragments de l'ADNc fut inséré dans un vecteur d'expression; un gène de fusion ainsi obtenu fut introduit dans $E$. coli. La protéine extraite des bactéries réagissait avec le sérum des sujets atteints d'une maladie d'Addison et eux seulement. Quelle peut être la signification physiopathologique de l'inactivation éventuelle de cette enzyme ? Dans la synthèse des stéroïdes, la voie de 17-hydroxylation conduit au cortisol, à la testostérone et aux œstrogènes; il existe une autre voie qui mène à l'aldostérone et à la progestérone. Une inhibition autoimmune de la 17- $\alpha$-hydroxylase pourrait donc expliquer une partie des symptômes, mais non la totalité. Chez certains malades, il se pourrait que des auto-anticorps dirigés contre d'autres
Tableau I

ENZYMES ANTÉRIEUREMENT RECONNUES COMME ANTIGĖNES DANS DES MALADIES AUTO-IMMUNES (D'après [1] et [4])

\begin{tabular}{|c|c|}
\hline Enzyme & Maladie \\
\hline $\begin{array}{l}\text { - Carboxypeptidase H } \\
\text { - Acide glutamique décarboxylase } \\
\text { (synthétisant le GABA) } \\
\text { - Cytochrome c P450 db-1 } \\
\text { - Peroxydase thyroïdienne } \\
\text { - } \mathrm{H}+\mathrm{K}+\mathrm{ATPase} \text { gastrique } \\
\text { - Myéloperoxydase } \\
\text { - Protéinase élastolytique }\end{array}$ & $\begin{array}{l}\text { Diabète type } 1 \\
\text { Diabète type } 1 \\
\text { Hépatite auto-immune type ॥ } \\
\text { Thyroïdite auto-immune } \\
\text { Gastrite auto-immune de l'anémie } \\
\text { pernicieuse } \\
\text { Glomérulonéphrite et vascularite } \\
\text { Granulomatose de Wegener avec } \\
\text { anticorps anti-neutrophiles }\end{array}$ \\
\hline
\end{tabular}


enzymes de la biosynthèse des stéroïdes soient impliqués. Ces anticorps auraient pu n'être pas détectés car les clones d'ADNc correspondants pourraient être absents de la banque de surrénales fotales criblée [5].

On ignore encore à ce jour si les anticorps anti-17 $\alpha$-hydroxylase sont causes de la maladie, ou bien sont secondaires à une atteinte auto-immune de la surrénale par des cellules $\mathrm{T}$ cytotoxiques. Il ne paraît de toute façon pas surprenant que des enzymes figurent parmi les auto-anticorps spécifiques d'organes ; il existe en effet plusieurs milliers de protéines-enzymes dont beaucoup ont une expression limitée à certains organes. On peut donc considérer [1] que les enzymes spécifiques d'organes cibles sont candidats à figurer comme auto-anticorps dans nombre de syndromes auto-immuns encore mystérieux. Si le travail de Krohn et al. est le premier à révéler des autoanticorps dans une maladie surrénale, d'autres ont déjà été décrits dans plusieurs maladies, et les exemples les mieux connus sont rappelés dans le Tableau I. L'existence d'auto-anticorps dirigés contre les enzymes physiologiquement importantes est appelée à prendre une place croissante dans la compréhension des maladies auto-immunes.

J.-C. D.

1. Editorial. Enzymes as autoantigens. Lancet $1992 ; 339: 779-80$

2. Ahonen P, Koskies S, Likki ML, Tillikainen A, Perheentupa J. The expression of autoimmune polygandular disease type 1 appears associated with several. HI_A-A antigens but not with HLA-DR. J Clin Endocrinol Metab $1988 ; 66$ : 1152-7.

3. MacLaren NK, Riley WJ. Inherited susceptibility to autoimmune Addison's disease is linked to human leukocyte antigens DR3 and/or DR4, except when associated with type 1 autoimmune polyglandular syndrome. J Clin Endocinol Metab 1986 ; 62 : 455-9.

4. Krohn K, Uibo R, Aavik E, Peterson P, Savilahti K. Identification by molecular cloning of an autoantigen associated with Addison' disease as steroid $17 \alpha$-hydroxylase. Lancet $1992 ; 339: 770-3$

5. Voutilainen R, Ilvesmäki V, Miettinen PJ Low expression of $3 \beta$-hydroxy-5-ene steroid dehydrogenase gene in human fetal adrenals in vivo. J Clin Endocin Metab 1991 ; 72 : 761-7.

$\mathrm{m} / \mathrm{s} \pi^{\circ} 5$, vol. 8 , mai 92

Pénétration d'enzymes lysoso-

Thérapie génique par transfert d'ADN in vivo chez le lapin hypercholestérolémique. Le lapin Watanabe est un modèle de l'hypercholestérolémie familiale par déficit en récepteur des LDL. Récemment, une correction partielle de ce déficit et une réduction prolongée de l'hypercholestérolémie ont été obtenues par greffe d'hépatocytes infectés ex vivo par un rétrovirus recombinant contenant l'ADNc du récepteur des LDL $\left(m / s n^{\circ} 2\right.$, vol. 8, p. 175). La même équipe, dirigée par J. M. Wilson (Ann Harbor, MI, USA), a parallèlement tenté de corriger le déficit par injection directe chez l'animal de l'ADN thérapeutique ciblé vers le foie. La méthode a consisté à coupler une asialoglycoprotéine, l'asialoorosomucoïde, à de la polylysine. Cette dernière joue le rôle de protéine se liant à l'ADN alors que l'asialoorosomucoïde est reconnue spécifiquement par les récepteurs hépatiques des asialoglycoprotéines, c'est-à-dire des glycoprotéines dont les acides sialiques ont été ôtés par action d'une sialidase. Après couplage à un plasmide contenant l'ADNc du récepteur des LDL, la préparation est directement injectée par voie intra-veineuse. Elle entraîne l'apparition extrêmement rapide dans le foie, de récepteurs de LDL, atteignant $2 \%$ à $4 \%$ de la concentration normale. Parallèlement, on note une diminution de $25 \%$ à $30 \%$ de la cholestérolémie, malheureusement très transitoire. Beaucoup de progrès sont donc encore nécessaires pour augmenter le niveau d'expression et, surtout, sa stabilité. D'ores et déjà, l'utilisation d'une préparation inerte d'ADN, c'està-dire d'un produit thérapeutique très sûr, pourrait avoir certaines indications, si une expression transitoire d'une protéine peut avoir des effets bénéfiques dans certaines affections aiguës.

[1. Wilson JM. J Biol Chem 1992'; 26 : 7963-7.] miales dans les neurones par conjugaison au fragment $\mathbf{C}$ de la toxine tétanique. On parvient aujourd'hui, grâce à des méthodes d'endocytose par l'intermédiaire d'un récepteur, à faire pénétrer des enzymes dans la cellule cible. Un exemple en est fourni par le traitement de la maladie de Gaucher (m/s $n^{\circ} 7$, vol. 7, p. 752). Dans le système nerveux, le problème est rendu plus difficile par l'existence de la barrière hématoencéphalique. De plus, lorsqu'on a pu circonvenir cette dernière, comme l'a fait Rattazzi (cité en [1]), en la perméabilisant, chez le chat atteint de déficit en hexosaminidase, l'enzyme ne pénétrait pas dans les neurones, rendant le traitement inefficace. La même équipe (Manhasset, NY, USA) a mis à profit l'existence, sur les neurones, de sites de liaison pour la toxine tétanique TT. Le fragment protéolytique atoxique $\mathrm{C}$ a été conjugué à de l'hexosaminidase [1]. Un mélange de cellules cérébrales de rat en culture fut mis à incuber avec cette préparation. Celle-ci pénétra dans les cellules neuronales; elle se montra capable d'abaisser la concentration en ganglioside GM2 (le produit qui s'accumule dans les cellules déficientes), dans des cellules corticales de chat atteint de déficit en hexosaminidase, montrant que l'enzyme parvient jusqu'aux lysosomes. La prochaine étape doit être l'essai in vivo chez les chats atteints de cette gangliosidose à GM2. Pour cela seront combinées les méthodes de perméabilisation de la barrière hématoencéphalique, ainsi qu'éventuellement la méthode hyperosmotique, déjà utilisée chez l'homme [2], avec l'emploi de l'hexosaminidase conjuguée. En cas de succès, on peut rêver d'une application ultérieure dans des maladies humaines...

[1. Dobrenis K, et al. Proc Natl Acad Sci USA 1992 ; 89 : 2297-301.]

[2. Neuwelt EA, et al. J Clin Oncol $1991 ; 9$ : 1580-90.] 
- Le rôle de la forme membranaire des $\operatorname{Ig} M$ précisé par recombinaison homologue. Les immunoglobulines peuvent être synthétisées sous une forme soluble ou membranaire en fonction d'un épissage alternatif, lui-même contrôlé par l'utilisation alternative de sites différents de polyadénylation. Des expériences antérieures avaient suggéré que la forme membranaire des immunoglobulines M (IgM) était essentielle au phénomène de l'" exclusion allélique ". Il s'agit du processus par lequel chaque lymphocyte $B$ ne possède qu'un seul allèle des chaînes lourdes d'immunoglobulines à contrôler de manière productive la synthèse d'anticorps. Tout se passe comme si le réarrangement productif (c'est-àdire aboutissant à la synthèse d'une protéine fonctionnelle) d'un gène de chaîne lourde, contemporain de la différenciation du lymphocyte pré-B en lymphocyte B, bloquait le réarrangement ultérieur de l'autre allèle et, en revanche, déclenchait le réarrangement des gènes de chaînes légères $\kappa$ suivi, si ce dernier n'a pas abouti à un agencement fonctionnel, du réarrangement des gènes codant pour les chaînes légères $\lambda$. Des expériences utilisant des souris transgéniques et des cellules lymphocytaires pré-B en culture avaient suggéré que c'était la synthèse de la forme membranaire de la chaîne lourde $\mu$ qui était responsable de l'exclusion allélique et, par conséquent, du déclenchement du réarrangement des gènes de chaînes légères. Kitamura et Rajewsky (Cologne, Allemagne) ont obtenu, en 1991 [1], des souris ayant subi une mutagenèse insertionnelle par recombinaison homologue au niveau de l'exon codant pour le segment transmembranaire de la partie constante de la chaîne $\mu$ de l'IgM. A l'état homozygote, ces souris ne produisent pas de cellules lymphocytaires B car leur développement est arrêté au stade pré-B, confirmant bien le caractère indispensable, pour la différenciation lymphocytaire B, d'un réarrangement productif des segements codant pour la forme membranaire de la chaîne $\mu$. Comme attendu, ces souris synthétisent extrêmement peu de chaînes $\kappa$ et possèdent, très majoritairement, des gènes $\kappa$ non réarrangés. Cependant, le blocage du réarrangement des segments $\kappa$ n'est pas complet, indiquant que le système de contrôle connaît certaines fuites. Les animaux hétérozygotes pour la mutagenèse insertionnelle ont un développement immunitaire normal. Si la synthèse de la forme membranaire de la chaîne $\mu$ est bien responsable du phénomène d'exclusion allélique, on doit s'attendre, chez ces animaux, à trouver des lymphocytes B ayant les deux allèles fonctionnels. Il est possible de distinguer les produits des deux allèles de chaînes lourdes d'immunoglobulines grâce à l'existence d'allotypes. Les souris hétérozygotes étudiées par Kitamura et Rajewsky ont subi l'événement de recombinaison homologue au niveau d'un allotype a et possèdent un allotype $b$ non modifié. De fait, un certain pourcentage de cellules double-positives sécrétant à la fois des IgM d'allotypes a et b est observé [2].

[1 Kitamura D, et al. Nature 1991 ; 350 : 423-6.]

[2 Kitamura D, Rajewsky K. Nature 1992 : 356 : 154-6.]

La potentialisation de longue durée s'applique aussi à des synapses inhibitrices. Les lecteurs de $\mathrm{m} / \mathrm{s}$ ont déjà souvent rencontré l'accroissement de l'efficacité d'une connexion synaptique, après stimulation conditionnante, que l'on appelle la potentialisation de longue durée (long term potentiation). Jusqu'à présent, celle-ci n'avait été rapportée que pour des synapses excitatrices dont la potentialisation aboutissait à l'augmentation de la réponse du neurone postsynaptique. Une équipe francoaméricaine animée par Henri Korn (Institut Pasteur, Paris) vient d'observer un phénomène similaire touchant, au contraire, une synapse inhibitrice liant le nerf VIII à la cellule de Mauthner controlatérale chez le poisson rouge [1]. Une stimulation tétanisante dans le nerf VIII provoque ainsi une modification de l'efficacité de la synapse aboutissant à une augmentation de l'inhibition induite par des stimulations ultérieures. La mise en évidence de la potentialisation de longue durée au niveau de contacts synaptiques de plus en plus nombreux permettait de penser que les synapses inhibitrices devaient aussi être concernées. Voilà qui est démontré. Il est classique d'associer les phénomènes de potentialisation de longue durée à des mécanismes impliquant des récepteurs du glutamate de type NMDA, quoique cela ait été discuté dans certains cas (voir $\mathrm{m} / \mathrm{s}, n^{\circ} 1$, vol. 7, p. 80). Il est peu probable que ces récepteurs jouent un rôle majeur dans les expériences rapportées ici, car la potentialisation de longue durée de la synapse inhibitrice dont le neurotransmetteur est la glycine - n'est pas bloquée par des antagonistes du glutamate. Il se confirme donc que la potentialisation de longue durée est un phénomène très répandu dans le système nerveux central ; les mécanismes en cause pourraient toutefois être beaucoup plus variés que cela pouvait paraître il y a encore peu de temps.

[1. Korn H, et al. Proc Natl Acad Sci USA 1992 ; 89 : 440-3.]

Tumorigénicité et instabilité génomique : deux modifications génétiques acquises récessives. L'instabilité du génome joue, très probablement, un grand rôle dans l'apparition et la progression des tumeurs. Elle explique, en effet, les multiples altérations génétiques nécessaires pour aboutir à une tumeur, ainsi que l'hétérogénéité cytogénétique très fréquente des cancers, avec leur lot de délétions, inversions, translocations, aneuploïdie, mutations 
ponctuelles et amplifications. L'amplification est, par exemple, un phénomène extrêmement fréquent dans les cellules cancéreuses [1]. Le schéma généralement admis de la progression tumorale comporte donc des mutations somatiques aléatoires, sélectionnées successivement lorsqu'elles entraînent un avantđge par rapport aux autres cellules : diminution de la mortalité ou augmentation de la prolifération. La liaison entre l'aptitude d'une cellule à amplifier certaines régions de son $\mathrm{ADN}$ et le caractère tumorigène est bien attesté par des expériences de Tlsdy et al. (Chapel Hill, NC, USA) : lorsque les cellules tumorales sont traitées par le PALA (N-[phosphonoacétyl]-L-aspartate), elles amplifient avec une grande fréquence le segment génique codant pour l'enzyme multifonctionnelle CAD (carbamyl phosphate synthétase, aspartate transcarbamylase et dihydro-orotase). La fréquence d'amplification est de $10^{-3}$ avec des cellules très tumorigènes, $10^{-6}$ avec des cellules immortalisées mais non tumorigènes, et pratiquemment nulle avec des cellules en culture primaire, diploïdes. Les auteurs ont alors créé des hybrides entre une lignée de cellules tumorigène et des fibroblastes diploïdes : les hybrides obtenus sont caractérisés par une diminution très considérable de la fréquence d'amplification du gène $C A D$ en présence de PALA. En revanche, ces hybrides conservent toute leur tumorigénicité [2]. Des expériences d'hybridation entre cellules somatiques avaient antérieurement démontré que le caractère tumorigène lui-même et l'immortalité pouvaient être supprimés dans des hybrides intersomatiques entre des cellules tumorales et des cellules normales. Cette suppression de la tumorigénicité ou de l'immortalité a pu être assignée à plusieurs chromosomes, formant différents groupes de complémentation. Les expériences rapportées maintenant montrent que l'aptitude à amplifier l'ADN est, elle aussi, un caractère récessif, indépendant de la $\mathrm{m} / \mathrm{s} n^{\circ} 5$, vol. 8, mai 92 tumorigénicité. Ce dernier point est important dans la discussion concernant les relations entre la cancérisation cellulaire et l'instabilité génétique. Si cette dernière était la conséquence de la transformation cellulaire, l'on pourrait attendre que les deux caractères évoluent tou jours de concert et que toute cellule tumorale conserve un génome instable. En revanche, si l'instabilité génétique est un phénomène précoce qui a présidé à l'apparition des multiples anomalies génétiques nécessaires à la cancérisation, on comprend que cette dernière, une fois établie, ne nécessite plus que persiste cette labilité du génome [2] Les résultats de Tlsty et al. favorisent donc ce second mécanisme. Ces auteurs montrent également que la labilité génique peut en réalité, être contrôlée par plusieurs mécanismes : la survenue de mutations ponctuelles, dont la fréquence est augmentée dans les cancers, semble en effet identique dans des cellules sensibles à l'amplification génique et dans des cellules insensibles. Ce dernier paramètre est analysé en mesurant le pourcentage de cellules devenant résistantes à l'ouabaïne, puisque les mécanismes de cette résistance semblent être, avant tout, des mutations ponctuelles qui abaissent l'affinité de la $\mathrm{Na}^{+} / \mathrm{K}^{+}$ATPase pour l'ouabaïne.

[1. Szepetowski $P$, et al. médecine/sciences $1991 ; 7$ : 14-21.]

[2. Tlsdy TD, et al. Science 1992 ; 255 : 1425-7.]

Vacciner avec de l'ADN. Le principe des immunisations, et par conséquent des vaccinations, est d'introduire dans un organisme possédant un système immunitaire, un antigène contre lequel va se développer une réaction immune. Cet antigène peut être un micro-organisme entier ou bien, de plus en plus souvent, une protéine ou un peptide purifiés. Cette procédure de purification peut être complexe, même lorsqu'un antigène protéique est obtenu par génie génétique. Dans ce cas, en effet, il faudra toujours isoler la protéine ou le peptide recombinant du milieu de culture ou du broyat du micro-organisme l'ayant synthétisé. Des chercheurs américains du Texas viennent de montrer qu'un ADN complémentaire pouvait être directement utilisé pour provoquer une réaction immune [1]. Le principe en est d'utiliser une méthode d'introduction de l'ADN dans les cellules que l'on appelle la biolistique. Il s'agit d'adsorber les molécules d'ADN à transférer sur des microparticules métalliques (le plus souvent en or) et de projeter celles-ci sur les cellules que l'on veut transfecter. Cette méthode est très utilisée, d'ores et déjà, en biotechnologie végétale puisque les voies traditionnelles d'introduction d'un ADN recombinant, par utilisation du plasmide Ti de la bactérie Agrobacterium tumefaciens, ne fonctionnent pas chez les monocotylédones. Il a cependant été montré que cette technique était également très prometteuse pour transférer de l'ADN dans des cellules animales et pouvait même être utilisée in vivo, sur des organismes entiers. Tang et al. ont ainsi "bombardé " la peau de jeunes souris, au niveau de l'oreille, avec des microprojectiles véhiculant un vecteur d'expression du gène de l'hormone de croissance. Trente des 34 souris ainsi traitées ont développé des anticorps spécifiques dans les semaines suivant l'inoculation. Une inoculation de rappel est possible. Afin de vérifier la généralité de cette approche, les auteurs ont également réussi à immuniser des souris contre de l' $\alpha 1$-antitrypsine. Un intérêt particulier de cette approche est qu'elle est de nature à stimuler fortement une réponse de type $T$, puisque l'antigène sera synthétisé par les cellules de l'organisme immunisé, comme cela se passe lors d'une infection virale.

[1. Tang DC, et al. Nature 1992 : 356 : 152-4.] 
Vers une étude rapide des interactions protéine-protéine. Si l'étude des interactions protéineprotéine a bénéficié largement des possibilités du génie génétique, la construction de nombreux mutants reste, cependant, une entreprise assez lourde. Pour s'affranchir de ces contraintes, Cull et al. (Palo Alto, CA, USA) [1] ont réussi à garder un lien physique entre un peptide et l'ADN qui porte l'information de sa séquence. Le but des auteurs était de trouver l'épitope responsable de la fixation de la dynorphine (un peptide opiacé) à un anticorps monoclonal spécifique : pour ce faire, les auteurs ont construit une banque de plasmides codant pour le gène lacl fusionné avec des dodécapeptides de séquence aléatoire. Ce même plasmide contient, en outre, deux sites de fixation pour la protéine lacI. Dans des bactéries, la protéine de fusion lacIpeptide produite se fixe à ses sites de reconnaissance sur le plasmide : cette liaison étant très stable (plusieurs jours) et à haute affinité $\left(\mathrm{Kd}<10^{-14}\right.$ $\mathrm{M})$, il est possible d'extraire le complexe ADN-lacI-peptide. Les complexes capables d'interagir avec l'anticorps anti-dynorphine fixé sur le support sont élués, et les plasmides ainsi sélectionnés sont réintroduits dans des bactéries. On peut répéter l'opération plusieurs fois pour enrichir la population de plasmides-peptides capables de réagir avec l'anticorps. Dans les expériences témoins, sans anticorps, on obtient un très faible bruit de fond (1\%). Après deux "tours ", deux tiers des clones bactériens réagissent avec un test ELISA : la séquence nucléotidique des plasmides montre la présence d'une séquence consensus présente dans la dynorphine. Les autres clones possèdent aussi la séquence consensus, qui n'est toutefois pas rencontrée dans les clones témoins : cela traduit une sensibilité supérieure au test ELISA, probablement en raison de la présentation multiple de l'antigène par les tétramères lacI. La possibilité de fusionner lacI (ou d'autres protéines se fixant à l'ADN) à des protéines sans perte de leurs propriétés pourra permettre à cette technique simple et puissante de rendre de grands services dans l'étude des interactions protéine protéine.

[1. Cull MG, et al. Proc Natl Acad Sci USA 1992 ; 89 : 1865-9.]

L L'altération du baroréflexe dans l'hypertension artérielle est en partie génétiquement déterminée. Le baroréflexe est constitué de récepteurs à l'étirement localisés dans le sinus carotidien et la crosse de l'aorte thoracique, et reliés au centre vasomoteur du tronc cérébral qui contrôle l'activité du système nerveux autonome. Ainsi le baroréflexe tend à éviter les fluctuations excessives de la pression artérielle. Ce système devrait ramener la pression artérielle à la normale en cas d'hypertension (HTA). La persistance d'une HTA témoigne donc d'une sensibilité diminuée du baroréflexe. Celle-ci est souvent interprétée comme la conséquence de l'HTA mais, dans certains modèles expérimentaux, elle peut précéder l'HTA. Enfin, des facteurs génétiques interviennent dans la pathogénie de l'HTA essentielle: concernent-ils la sensibilité du baroréflexe ? Parmer, Cervenka et Stone (San Diego, CA, USA) ont abordé ce problème en étudiant 40 hypertendus essentiels non traités [27 ayant des antécédents familiaux d'HTA $(\mathrm{FH}+), 13$ n'en ayant pas $(\mathrm{FH}-)]$ et 20 sujets normotendus dont $50 \%$ étaient $\mathrm{FH}+$ et $50 \% \mathrm{FH}-$. Le baroréflexe a été exploré en étudiant le ralentissement cardiaque induit par la phényléphrine (qui augmente la pression artérielle) et l'accélération produite par le nitrite d'amyle (qui entraîne une baisse de la pression artérielle) [1]. Chez les sujets $\mathrm{FH}+$, hypertendus ou normotendus, la sensibilité du baroréflexe est diminuée. Ces résultats suggèrent que l'altération du baroréflexe pourrait être un des éléments qui détermine la prédis- position génétique à l'HTA familiale. En outre, la réponse au nitrite d'amyle diminue avec l'âge chez les sujets $\mathrm{FH} \mathrm{-} \mathrm{(problablement} \mathrm{du} \mathrm{fait}$ d'une diminution de la distensibilité artérielle), mais non chez les sujets $\mathrm{FH}+$, comme si ceux-ci développaient plutôt l'altération génétiquement déterminée du baroréflexe, indépendamment des effets du vieillissement.

[1. Parmer RJ, et al. Circulation 1992 ; 85 : 497-503.]

Une protéine de l'émail dentaire est codée à la fois par les chromosomes $\mathbf{X}$ et $\mathbf{Y}$. Amélogénines et énamélines sont les deux classes principales de protéines dans la matrice extracellulaire de l'émail dentaire. L'émail précoce contient des amélogénines, riches en proline, remplacées plus tard par des énamélines acides. A partir de clones (isolés depuis 1985), on a pu assigner le gène de l'amélogénine [1] au chromosome $\mathrm{X}$ chez la souris, et chez l'homme en Xp22.1-p22.3, et lui rattacher une maladie génétique affectant la formation de l'émail; une délétion de ce gène a été repérée dans une famille [2]. A côté de ce gène $A M G X$, on a pu en repérer un autre dans la région péricentrique du chromosome Y, appelé $A M G Y$. On ignorait toutefois s'il était fonctionnel. Un ADNc fut isolé à partir de bourgeons dentaires de fotus masculins et féminins de 20 à 25 semaines. On a pu ainsi cloner séparément des ADNc provenant des gènes de $\mathrm{X}$ et de $\mathrm{Y}$. On a pu voir [3] que les transcrits présentaient des différences (793 bp codantes pour $A M G X, 802$ pour $A M G Y$ ), ainsi que les protéines mûres (175 acides aminés contre 176); il semble que dans les deux cas il existe un peptide signal de 16 acides aminés. Des formes mineures de transcrits font penser à des épissages alternatifs. Les auteurs ont démontré que le gène $Y$ est bien transcrit, bien qu'à un niveau 10 fois inférieur à celui de 
l'X, et qu'il code pour une protéine fonctionnelle, tout au moins à ce stade fotal. Les gènes identifiés jusqu'à présent à la fois sur $\mathrm{X}$ et sur $Y$ échappent à l'inactivation de l'X. Dans le cas de l'amélogénine, l'image en mosaïque de l'émail chez les femmes hétérozygotes pour le déficit génétique, amelogenesis imperfecta, fait penser que l'inactivation se produit. S'il en est bien ainsi, le système AMGX/AMGY serait unique en ce que, potentiellement, les hommes fabriqueraient plus de produit que les femmes. Une bien petite différence en vérité...

[1. Lau EC, et al. Genomics $1989 ; 4$ : 162-8.]

[2. Lagerstrom M, et al. Genomics $1991 ; 10$ : 971-5.]

[3. Salido EC, et al. Am J Hum Genet 1992 ; 50 : 303-16.]

Le TNF $\alpha$, une protéine pouvant s'insérer dans la membrane cellulaire et former des pores perméables aux ions. Le numéro de mai de médecine/sciences ( ${ }^{\circ} 5$, vol. 8 ) contient plusieurs contributions consacrées à des peptides à effet antimicrobien, chez les vertébrés et les invertébrés [1-3]. La plupart de ces peptides anti-infectieux, dont certains sont actifs sur des cellules eucaryotes, agiraient en s'intercalant dans la bicouche lipidique des membranes cellulaires, y formant des canaux ioniques peu sélectifs et dépendants du voltage. La dissipation de la différence de potentiel membranaire qui s'ensuivrait serait responsable de l'effet cytolytique de ces substances. Des chercheurs de Los Angeles (CA, USA) rapportent maintenant que le $\mathrm{TNF} \alpha$ (tumor necrosis factor $\alpha$ ) pourrait, lui aussi, former de tels canaux ioniques qui expliqueraient certains de ses effets physiologiques, notamment son effet cytotoxique direct [4]. La molécule de $\mathrm{TNF} \alpha$ mûre est un trimère compact de $51 \mathrm{kDa}$ dont les sous-unités se présentent comme des cylindres $\beta$ anti-parallèles, ménageant $\mathrm{m} / \mathrm{s} n^{\circ} 5$, vol. 8 , mai 92 entre eux un long canal. Cette molécule est capable de provoquer une diminution rapide du potentiel de membrane, associée à une augmentation de la perméabilité pour le sodium. L'effet canal de l'insertion du TNF $\alpha$ dans la membrane plasmique peut être confirmé par des expériences d'électrophysiologie. Le canal/TNF serait dans une conformation ouverte à bas $\mathrm{pH}$ expliquant l'augmentation de la cytotoxicité du $\mathrm{TNF} \alpha$ aux $\mathrm{pH}$ acides. La découverte de substances susceptibles de moduler la perméabilité des pores formés par le $\mathrm{TNF} \alpha$ permettrait peut-être d'atténuer les conséquences défavorables de cette cytokine, par exemple en cas de choc septique ou de paludisme grave.

[1. Pattus F. médecine/sciences 1992 ; 8 : 420-2.]

[2. Hoffmann J, et al. médecine/sciences $1992 ; 8$ : 432-9.]

[3. Nicolas $\mathrm{P}$, et al. médecine/sciences $1992 ; 8$ : 423-31.]

[4. Kagan PL, et al. Science 1992 ; $255: 1427-30$.]

Clonage de l'ADNc codant pour un récepteur du TGF $\beta$. Le TGF $\beta$ a de nombreuses fonctions biologiques, activant l'angiogenèse, bloquant ou stimulant la différenciation de certaines cellules et inhibant très efficacement la prolifération de types variés de cellules épithéliales. Des méthodes biochimiques ont permis de décrire trois types de récepteurs du TGF $\beta$ désignés types I, II et III. L'équipe de H. F. Lodish avait déjà cloné, par expression dans des cellules COS, un ADNc codant pour le récepteur de type III qui s'est révélé être un protéoglycane [1]. En utilisant la même méthode, H. Y. Lin, également du laboratoire de $\mathrm{H}$. F. Lodish au MIT (Cambridge, MA, USA), est maintenant parvenu à isoler un $\mathrm{ADNc}$ codant pour le récepteur de type II qui est une protéine de 565 acides aminés à un seul passage trans-membranaire et dont une grande région carboxy-terminale cytoplasmique possède un domaine de type protéine kinase spécifique des résidus sérines et thréonines [2]. A ce titre, le récepteur de type II du TGF $\beta$ montre de nettes similitudes avec celui de l'activine, un peptide hormonal sécrété par l'ovaire et initialement décrit pour ses propriétés de stimulation de la sécrétion hypophysaire de FSH (folliclestimulating hormone) $\left(\mathrm{m} / \mathrm{s} n^{\circ} 8\right.$, vol. 2, p. 466). On sait maintenant que l'activine intervient également dans bien d'autres phénomènes, notamment l'induction de la formation du mésoderme au cours de l'embryogenèse. La similitude notée des récepteurs n'est pas très étonnante compte tenu des analogies de séquences entre le TGF $\beta$ et l'activine. Il est peut-être significatif de trouver ici un récepteur d'un facteur inhibiteur de prolifération, doté d'une très probable activité de sérine/thréonine kinase, alors que les récepteurs de facteurs de croissance sont, très souvent, des tyrosine kinases. Peut-être cette différence fonctionnelle est-elle le reflet de la modulation positive (via les tyrosine kinases) et négatives (via ce récepteur sérine/thréonine kinase) de mêmes cibles appartenant au système de stimulation de la prolifération cellulaire. Le rôle respectif des différents récepteurs du TGF $\beta$ n'est pas connu. On peut suggérer que les récepteurs de type II, et peut-être ceux de type I, coopèrent avec les récepteurs de type III pour parvenir à une meilleure transduction du signal. Une coopération de même type entre des récepteurs protéine kinase (ici tyrosine kinase) à haute affinité et des récepteurs à basse affinité de type protéoglycanes a déjà été décrite dans le cas des récepteurs du FGF (fibroblast growth factor) [3].

[1. Wang XF, et al. Cell 1991; 67 : 767-805.

[2. Lin HY. Cell $1992 ; 68$ : 775-85.]

[3. Courtois Y. médecine/sciences 1990 ; 6 : 674-9.] 
La glycoprotéine gp170, produit du gène MDR1 (multidrug resistance) est un canal chlore contrôlé par le volume cellulaire. Les gènes MDR (multidrug resistance) codent pour des glycoprotéines (gp170) qui pompent hors de la cellule des substances hydrophobes, notamment des médicaments divers utilisés pour leurs propriétés anti-mitotiques [1]. Ces molécules appartiennent à une plus large famille dite $\mathrm{ABC}$ (ATP-binding cassette), comportant toute une série de transporteurs liant et utilisant l'ATP. Parmi les membres de cette famille, on trouve des transporteurs bactériens, le probable transporteur de peptides se liant aux molécules de classe 1 du complexe majeur d'histocompatibilité et la molécule CFTR, canal chlore contrôlé par l'AMP cyclique défectueux chez les malades atteints de mucoviscidose. La molécule CFTR se trouve normalement au pôle apical de très nombreuses cellules épithéliales sécrétrices. Ces cellules contiennent d'autres types de canaux chlore, fonctionnellement réglés par le calcium et le volume cellulaire. Or la gp170 est abondante dans de nombreux types de cellules épithéliales sécrétrices. Deux équipes anglaises viennent de démontrer que ce produit du gène MDR1 est un canal chlore dépendant de l'ATP et contrôlé par le volume cellulaire, correspondant par conséquent à une classe de canal qui avait été fonctionnellement caractérisée précédemment mais dont le substratum moléculaire n'était pas connu [2]. Ces résultats sont importants et résolvent, probablement, une énigme sur laquelle nous avons attiré l'attention de nos lecteurs plusieurs fois : étant bien entendu que le rôle fonctionnel du gène MDR1 n'est pas d'expulser de la cellule des médicaments anticancéreux, quelle peut bien être sa fonction normale ? Cette révélation que la gp170 est, à la fois, un transporteur de substances hydrophobes et un canal chlore soulève, cependant, immédiatement une autre question : la molécule CFTR, qui est un canal chlore dont l'ouverture est stimulée aussi, la propriété de transporter d'autres molécules?

[1. Marie JP. médecines/sciences 1990 ; 6 : 443-8.]

[2. Valverde MA, et al. Nature 1992 ; 355 : 830-3.]

La mutation Trembler de la souris est due à une mutation ponctuelle du gène de la protéine périphérique de la myéline (PMP-22). La souche de souris mutante dite Trembler (en raison, évidemment, de son comportement) est apparue spontanément à la suite d'une mutation dont la transmission est autosomique dominante. Du point de vue histopathologique, cette mutation s'accompagne d'un défaut majeur de la myélinisation des nerfs périphériques et d'une prolifération continue des cellules supposées remplir ce rôle de myélinisation, les cellules de Schwann. La mutation Trembler a été localisée sur le chromosome 11 de la souris. Eric Shooter et son équipe (Stanford, CA, USA) ont récemment décrit une protéine spécifique de la myéline périphérique (PMP-22) [1] qui apparaissait comme un bon candidat à la mutation. En effet, en l'absence de défaut de myélinisation des axones du système nerveux central, on pouvait supposer que la mutation devrait intéresser une protéine de ce genre, que l'on ne trouve que dans les nerfs périphériques. La même équipe confirme aujourd'hui son hypothèse en démontrant que : (1) le gène codant pour PMP-22 est localisé sur le chromosome 11 de la souris et que (2) les ADNc pour PMP-22 obtenus par PCR chez Trembler présentent une mutation ponctuelle substituant un acide aspartique à une glycine dans une des régions transmembranaires de la protéine. Il existe plusieurs maladies génétiques chez l'homme qui se traduisent par des déficits myéliniques de localisation périphérique. La plus connue est la neuropathie hypertrophique héréditaire (Charcot-MarieTooth, type 1a). Les auteurs proposent PMP-22 comme gène candidat pour cette affection et suggèrent d'en rechercher l'équivalent humain sur le bras court du chromosome 17 (p12-p11.2) où l'on a localisé la mutation responsable de la neuropathie...

11. Welcher A, et al. Proc Natl Acad Sci USA 1991; 88 : 7195-9.]

[2. Suter U, et al. Nature $1992 ; 356$ : 241-4.]

Un nouveau modèle d'hypertension créé chez la souris par transgénèse. Le système rénineangiotensine comporte trois composants majeurs, l'angiotensinogène, la rénine et l'enzyme de conversion qui jouent un rôle très important dans la régulation de la pression artérielle et de l'homéostasie des électrolytes dans l'organisme. Les groupes de Mullins (Heidelberg, RFA) [1] et d'Ohkubo (Kyoto, Japon) avaient respectivement créé en 1990 [2] des rats transgéniques exprimant le gène Ren-2 et des souris exprimant soit la rénine, soit l'angiotensinogène, soit ces deux protéines simultanément. Les rats Ren-2, caractérisés par de très faibles concentrations plasmatiques de rénine et d'angiotensine II (produit de transformation de l'angiotensine I par l'enzyme de conversion), et les souris double-transgéniques développaient une hypertension d'importance variable, alors que les souris simple transgéniques, exprimant soit la rénine, soit l'angiotensinogène n'avaient pas d'hypertension malgré une concentration élevée de l'une ou l'autre de ces protéines dans le plasma. Mullins et al. viennent de créer un modèle complémentaire en injectant le gène codant pour l'angiotensinogène de rat dans l'embryon de souris [3]. Les trois lignées obtenues diffèrent, du fait de l'influence des séquences jouxtant les sites d'intégration, par le niveau d'expression et la localisation de l'expression du transgène. L'une des lignées exprime le transgène dans le foie et le cerveau, expression qui suit celle du gène endogène. L'autre lignée exprime le transgène dans le foie et le cerveau mais de façon ectopique en ce qui concerne ce dernier. Dans les deux cas, les protéines circulantes du 
système rénine-angiotensine sont en concentration élevée dans le plasma mais seuls les animaux de la lignée surexprimant le transgène de façon non ectopique dans le cerveau développent une hypertension. Ce résultat laisse penser qu'une distribution correcte de l'expression du transgène dans le cerveau est nécessaire pour que survienne l'hypertension, alors que la simple élévation des concentrations plasmatiques des protéines du système rénine-angiotensine, est inefficace.

[1. Mullins J, et al. Nature 1990 ; 344 : 541-4.]

[2. Ohkubo H, et al. Proc Natl Acad Sci USA 1990 ; 87 : 5133-7.]

[3. Kimura S, et al. EMBO J 1992 ; $11: 821-7$.

Attention au ballon ! Échapper à un objet évoluant rapidement sur une trajectoire de collision est une tâche auquel le système nerveux de tous les animaux doit faire face avec célérité et précision. Le point de départ de la décision d'évitement doit se situer, à l'évidence, dans la reconnaissance d'un stimulus visuel comme une menace directe, donc dans une évaluation précise de la trajectoire et du moment de l'impact prévisible. Wang et Frost (Queens University, Canada) viennent d'enregistrer dans un noyau mésencéphalique, chez le pigeon, des neurones dont la responsabilité paraît entière dans ces phénomènes de reconnaissance [1], donc dans le déclenchement des comportements d'évitement. Ces neurones, situés dans la région postérieure et dorsale du nucleus rotundus, présentent une activité brutale et massive lorsqu'un ballon de football s'apprête à heurter la tête du pigeon, en fait très précisément 1 seconde $(0,8$ à 1,4$)$ avant l'impact. Cette activation ne dépend ni de la taille du ballon, ni de l'angle de sa trajectoire, ni même de sa vitesse. Les seuls paramètres reconnus sont le risque de collision et sa latence. Les auteurs ne cherchent pas à identifier les mécanismes de l'activation neuronale, mais on peut suggérer que, n'étant pas directement dépendants de l'une des caractéristi- ques véhiculées par le système de transmission visuel rétino-tectale, ils proviennent d'une intégration multisensorielle et, très vraisemblablement, d'un apprentissage. Quelques neurones spécialisés transformeraient ainsi les informations sur la trajectoire du stimulus et celle du corps, de même que leurs vitesses relatives, en données temporelles enclenchant des processus comportementaux à la fois très divers et stéréotypés. Petite note pour rassurer les éventuels défenseurs de pigeons, toutes ces expériences ont été réalisées, chez l'animal anesthésié, à l'aide d'une simulation de ballon sur ordinateur!

[1. Wang Y, Frost BJ. Nature 1992 ; 356 : 236-8.]

La paramyotonie congénitale, comme la paralysie périodique hyperkaliémique, est due à une mutation du canal sodium des muscles squelettiques [1]. La paramyotonie congénitale est une affection dont la symptomatologie est sensiblement différente de celle de la paralysie périodique hyperkaliémique ( $\mathrm{PPH})$ caractérisée par une myotonie induite par le froid. Les deux maladies sont cependant dues à la mutation du même gène, quoique dans des domaines différents. Dans la paramyotonie congénitale, deux mutations ont été notées dans la boucle cytoplasmique comprise entre les troisièmes et quatrièmes segments transmembranaires alors que les lésions dans la $\mathrm{PPH}$ étaient localisées dans des segments transmembranaires. Ces résultats indiquant que des affections différentes peuvent être les conséquences de la mutation d'un même gène n'ont rien de particulièrement étonnant et connaissent de très nombreux antécédents pour ce qui est des mutations des gènes de globine ou d'enzymes, notamment érythrocytaires.

[1. Fontaine B. médecine/sciences 1992; 8 : 41-5.]

[2. McClatchey AL, et al. Cell 1992 ; 68 : 769-74.]

Progression tumorale de tumeurs cérébrales et mutations de p53. L'équipe de B. Vogelstein (Baltimore, MD, USA) vient de rapporter l'étude des mutations de l'antigène p53 dans des couples de prélèvements issus de tumeurs cérébrales étudiées précocement et tardivement au cours de l'évolution. Dans sept cas, la tumeur était d'emblée de haut grade (glioblastome) alors que trois fois elle avait évolué d'un stade de bas grade (astrocytome) à une forme plus maligne. Le gène $p 53$ était muté trois fois au niveau des prélèvements initiaux de glioblastomes, quatre fois au niveau des prélèvements plus tardifs. En revanche, aucun astrocytome (prélèvements initiaux) ne comportait de mutations de $p 53$ alors que celleci était retrouvée lorsque ces tumeurs de faible malignité avaient évolué vers un glioblastome [1]. Un examen plus attentif des tumeurs peu malignes retrouvait cependant, à leur niveau, une sous-population cellulaire possédant déjà la mutation. Il est probable que celle-ci possède un avantage prolifératif conduisant à son expansion clonale, phénomène essentiel de la progression tumorale.

[1. Sidransky D, et al. Nature 1992 ; 355 : 846-7.].

L'oncogène ras : signal mitogène ou signal de mort ? L'“ activation " par mutation ponctuelle des oncogènes de la famille ras $\left(\mathrm{H}^{-}, \mathrm{K}^{-}\right.$ et $\mathrm{N}^{-}$) intervient à un stade précoce de la tumorigenèse dans plusieurs types de cellules humaines, notamment celles de l'épithélium du côlon, de la thyroïde et du pancréas. Dans le cas de la thyroïde, le rôle clé de ras a été récemment confirmé par deux équipes anglaises de Cardiff (dirigées par D. Wynford-Thomas), qui ont montré qu'un gène ras muté, introduit dans des cellules thyroïdiennes normales au moyen d'un vecteur rétroviral, engendre une prolifération dont les caractéristiques rappellent in vitro celles d'un adénome (tumeur bénigne) in vivo [1, 2]. En poursuivant cette voie de recherche, ces auteurs ont maintenant obtenu un résultat tout à fait inattendu, qui pourrait avoir des retombées très importantes sur le plan thérapeutique

.

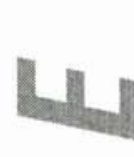


aussi bien que fondamentales. Ils ont trouvé [2] que la prolifération induite par l'expression de ras muté ne se produit pas si les cellules thyroïdiennes sont traitées en même temps par un phorbol ester (tetradecanoylphorbol acetate, TPA). Par ailleurs, non seulement le TPA inhibe la croissance stimulée par ras, mais il entraîne aussi la mort rapide de ces cellules sans (il faut le souligner) aucun effet toxique sur des cellules normales (qui n'expriment qu'un ras normal). Cet effet toxique du TPA en présence de ras muté a été démontré par la suite dans des lignées de cellules thyroïdiennes immortalisées par l'antigène $T$ du virus SV40 et qui contiennent un ras muté dont l'expression est inductible ( $T$. Dawson et al., en préparation). Puisque ces lignées (à la différence des cellules primaires) poussent indépendamment de l'expression de ras, cette expérience prouve que l'effet toxique n'est pas simplement une fonction de la prolifération induite par ras muté dans les cellules primaires. Par ailleurs, on a réussi à l'aide de TPA à modifier l'action prolifératrice de ras muté en un signal de mort cellulaire. Que la perception d'un signal biochimique puisse changer selon le " terrain " cellulaire a déjà été reconnu lors du développement lymphocytaire [3]. Ces expériences représentent cependant la première mise en évidence de ce phénomène dans une cellule épithéliale et en réponse à un oncogène activé. L'intérêt thérapeutique est évident, d'autant plus que les voies biochimiques impliquées dans l'action du TPA (notamment la famille d'enzymes, protéine kinase $C$ ) représentent une cible particulièrement commode vis-à-vis de laquelle plusieurs substances actives ont déjà été développées. [1. Lemoine NR, et al. Oncogene 1990 ; 5 : 1833-7.]

[2. Bond J, et al. Carcinogenesis 1992 ; 5 : 129-35.]

[3. Golstein P. médecine/sciences 1991 ; $7:$ 681-6.] 